Andreas Hofmann/Wolfgang Wessels ${ }^{1}$

\title{
Tektonische Machtverschiebungen - die Krise als Auslöser und Verstärker des institutionellen Wandels
}

Wer in den letzten Jahren die Bemühungen um die Bewältigung der nicht enden wollenden Krise der Eurozone mitverfolgte, hat verlernt überrascht zu sein. Was vor den Krisenjahren als undenkbar galt, haben die Regierungen der Mitgliedstaaten und die Organe der EU in den letzten Jahren als unvermeidbar deklariert: die Schaffung eines Rettungsfonds, der gemeinschaftlich garantierte Kredite in dreistelliger Milliardenhöhe für Mitgliedstaaten der Eurozone bereitstellt, eine zunehmend engere Überwachung nationaler Haushalte und die vertragliche Verpflichtung, die Begrenzung von Staatsdefiziten in nationalen Verfassungen zu verankern, unbegrenzte Ankäufe nationaler Staatsanleihen von Seiten der Europäischen Zentralbank (EZB) auf dem Sekundärmarkt, Überwachungsmissionen der Kommission und der EZB in Ländern, die von der bereitgestellten Finanzhilfe Gebrauch gemacht haben, die Schaffung einer »Bankenunion« und die Einführung einer Finanztransaktionssteuer. Staats- und Regierungschefs im Europäischen Rat sahen sich mit großer Regelmäßigkeit genötigt zu betonen, die gemeinsame Währung sei unumkehrbar und man werde alles Notwendige zu ihrer Rettung tun. $\mathrm{Zu}$ diesem Zweck wurde schließlich eine neue Institution geschaffen: der Euro-Gipfel verstetigt nach vertraglich vereinbarten Regeln die sich seit den frühen Krisenjahren häufenden ad hoc Treffen der Staats- und Regierungschefs der Eurozone. Zusätzlich zu den bereits beschlossenen Maßnahmen führen die Regierungen der Mitgliedstaaten und die Organe der EU eine vehemente Diskussion um noch zu tätigende Schritte, wie etwa die Einrichtung eines gemeinschaftlichen Schuldentilgungsfonds, eines separaten Budgets für die Eurozone und die Schaffung einer europäischen Fiskalverwaltung. Die Hoffnung auf eine »konstitutionelle Stabilität « nach dem Inkrafttreten des Vertrags von Lissabon ${ }^{2}$ ist somit in weite Ferne gerückt.

Existenzkrisen sind Auslöser und Verstärker von grundsätzlichen Verschiebungen inter-institutioneller Beziehungen in allen politischen Systemen. Für die Europäische Union haben viele Kommentatoren Krisen als wesentliche Ursache von bedeutsamen Integrationsschritten identifiziert. ${ }^{3}$ Nach einem Jahrzehnt, in dem die Mitgliedstaaten bereits mit dem Verfassungsvertrag und dessen Nachfolger, dem Vertrag von Lissabon, sowie den Erweiterungen von 2004 und 2007 die konstitutionelle und geographische Architektur der Union neu gestalteten, hat die Krise der Wirtschafts- und Währungs-

1 Die Autoren danken Hanna-Lisa Hauge und Leonie Völker für ihre vielfältige Zuarbeit.

2 Vgl. Europäischer Rat, Schlussfolgerungen des Vorsitzes, 14. Dezember 2007.

3 Vgl. Ludger Kühnhardt (Hg.), Crises in European Integration: Challenges and Responses, 1945-2005, Oxford/New York 2009. 
union die Regierungen der Mitgliedstaaten und die Organe der EU nun vor gänzlich neue und gravierende Herausforderungen gestellt. Bei den Krisenbewältigungsmaßnahmen beobachten wir eine Reihe von fundamentalen Verschiebungen im institutionellen Gleichgewicht, die das vertikale Verhältnis von Mitgliedstaaten zur Union sowie die horizontale Balance des Einflusses einzelner Organe in der EU Architektur nachhaltig verändern. ${ }^{4}$ Beide Aspekte stehen im engen Wechselverhältnis. Ziel dieses Artikels ist es, diesen Verschiebungen im institutionellen Rahmen der Europäischen Union im Zuge der Krise nachzugehen. Der Artikel stellt dabei drei zentrale Fragen, die unsere Analyse strukturieren:

1. Wie verändern die Krisenbewältigungsmaßnahmen die (vertikale) Zuständigkeitsverteilung zwischen den Mitgliedstaaten und der Union?

2. Wie verändern die Krisenreaktionen das (horizontale) Gleichgewicht der Aufgabenverteilung zwischen den Akteuren in der institutionellen Architektur der EU?

3. Welche Quellen demokratischer Legitimation beanspruchen die Entscheidungsträger für die neuen Maßnahmen?

Unsere Analyse konstatiert dabei die Fortsetzung bereits bestehender Trends sowie einige überraschende Zäsuren. Als Antwort auf die Frage der vertikalen Kompetenzverteilung haben die Mitgliedstaaten wesentliche Instrumente auf die EU-Ebene verlagert. Nationale und EU-Verfahren werden zunehmend verknüpft und fusioniert. ${ }^{5} \mathrm{Zu}$ den »offensichtlichen Gewinnern« der Krisenjahre kann zweifelsohne der Europäische Rat gezählt werden, der seine Position als zentrale Leitinstanz in allen konstitutionellen Fragen weiter ausgebaut hat, sowie auch die Europäische Zentralbank, der von den Markteilnehmern als einziger Institution das Vertrauen entgegengebracht wurde, nicht nur über umfangreiche Ressourcen zur Lösung finanzieller Problemlagen zu verfügen, sondern auch über die notwendige Effizienz der Entscheidungsprozesse - besonders dann, wenn prompte Reaktionen unerlässlich schienen. Aufgrund ihres Unvermögens, die tagespolitische Diskussion wesentlich zu beeinflussen, wurde die Europäischen Kommission oft zu den »Verlierern« der Krisenjahre gezählt; aber auch ihr kann ein Zuwachs exekutiver Kompetenzen attestiert werden. Die Kommission ist in alle in den letzten Jahren beschlossenen entscheidenden Maßnahmen zur Überwachung nationaler Wirtschafts- und Fiskalpolitik an zentraler Stelle eingebunden. Eine Zäsur gegen bisherige Integrationstrends stellt dagegen die weitgehende Umgehung des Europäischen Parlaments (EP) dar. Weder haben ihm die Staats- und Regierungschefs eine zentrale Position in der Formulierung der Krisenreaktionen eingeräumt, noch wurden seine Kompetenzen durch die beschlossenen Maßnahmen ausgebaut. Vielmehr basiert die demokratische Legitimation nahezu der gesamten Krisenreaktionen auf nationalen Institutionen. Manche nationalen

4 Vgl. auch Wulf Reiners / Wolfgang Wessels, »Rivalität und Gleichgewicht in der institutionellen Architektur der EU« in: Werner Weidenfeld / Wolfgang Wessels (Hg.), Jabrbuch der Europäischen Integration 2012, Baden-Baden 2012, S. 45-52.

5 Vgl. Wolfgang Wessels, »An Ever Closer Fusion? A Dynamic Macropolitical View on Integration Processes « in: Journal of Common Market Studies 35, Nr. 2 (1997), S. 267-299; Wolfgang Wessels, »The Constitutional Treaty: Three Readings from a Fusion Perspective «, Journal of Common Market Studies 43, Annual Review (2005), S. 11-36.

ZfP 60. Jg. 2/2013 
Parlamente konnten durch die Notwendigkeit der Ratifikation zentraler Krisenmaßnahmen zusätzliche Kompetenzen erringen. Im Gegenzug beschränken aber EU-Regeln in Zukunft ihre Hoheit über nationale Fiskalpolitik in größerem Maße als zuvor.

\section{Die Dilemmata der Krisenbewältigung}

Wir gehen bei unserer Analyse der Krisenbewältigungsmaßnahmen davon aus, dass die Staats- und Regierungschefs der Mitgliedstaaten sich in der Beantwortung der durch die Krise neu aufgeworfenen (oben geschilderten) Fragen drei zentralen Dilemmata gegenüber sahen (siehe Übersicht 1), die ambivalente Einstellungen und Verhaltensmuster der Mitgliedstaaten bei der Gestaltung des EU-Systems seit Beginn des Integrationsprozesses prägen und auch - oder gerade - im Verlauf der Krise wesentliche Veränderungen des institutionellen Rahmens der EU und dessen Komplexität erklären helfen. ${ }^{6}$

Übersicht 1: Das dreifache Dilemma

\begin{tabular}{|l|c|c|}
\hline & Europäische Ebene & Nationale Ebene \\
\hline Ebenendilemma & Problemlösungsinstinkt & Souveränitätsreflex \\
\hline Entscheidungsdilemma & Effizienzsuche & Letztentscheidungsvorbehalt \\
\hline Legitimitätsdilemma & eigenständig europäisch & abgeleitet national \\
\hline
\end{tabular}

Quelle: Eigene Darstellung.

Das Ebenendilemma zeigt sich darin, dass Staats- und Regierungschefs der Mitgliedstaaten einerseits betonen, das »Friedens und Wohlstandsversprechen « der europäischen Einigung nur durch »gemeinsame Anstrengungen « verwirklichen zu können.? Im Zuge der Krise sehen sie sich mit großer Regelmäßigkeit mit Herausforderungen konfrontiert, die scheinbar nur durch konzertierte gemeinsame Reaktionen bewältigt werden können. Die Regierungen demonstrieren im gemeinschaftlichen Handeln einen »Problemlösungsinstinkt«, der zunehmend das EU-System als bestmögliche Problemlösungsebene identifiziert. Andererseits zeigen die Regierungen jedoch eine fortdauernde, als »Souveränitätsreflex« zu bezeichnende Zurückhaltung, zentrale Kompetenzen nationaler Wirtschafts- und Haushaltspolitik auf die europäische Ebene zu übertragen.

Mit der Wahl einer geeigneten Problemlösungsebene erschließt sich ein neues Dilemma. Auf der einen Seite streben die Regierungen der Mitgliedstaaten möglichst effiziente Entscheidungsfindungsmechanismen an, um kurzfristig und überzeugend auf Krisensymptome reagieren zu können. Dieses Bestreben kann als »Effienzsuche« bezeichnet werden. Gleichzeitig haben die Staats- und Regierungschefs gerade bei sensiblen Fragen der nationalen Budgethoheit wiederholt demonstriert, hier nationale »Letztentschei-

6 Vgl. Andreas Hofmann / Wolfgang Wessels, »Der Vertrag von Lissabon - eine tragfähige und abschließende Antwort auf konstitutionelle Grundfragen? « in: integration, Nr. 1 (2008), S. 3-20.

7 Angela Merkel, Regierungserklärung von Bundeskanzlerin Merkel zum Europäischen Rat am 13. und 14. Dezember 2012 in Brüssel, 13. Dezember 2012. 
dungsvorbehalte in Form von Einstimmigkeitserfordernissen aufrechtzuerhalten und damit die Vetomacht jedes Mitglieds zu konservieren.

Schließlich stehen die Regierungen der Mitgliedstaaten vor der Herausforderung, die gefundenen Maßnahmen vor der weitgehend skeptischen und nach wie vor vorwiegend national konstituierten Öffentlichkeit rechtfertigen zu müssen. Historisch haben sich die Träger konstitutioneller Entscheidungen in der EU auf zwei unterschiedliche Quellen der Legitimation berufen: einer eigenständig europäischen, oftmals mittels einer Stärkung der Mitwirkungsrechte des Europäischen Parlaments, sowie einer abgeleitet nationalen mit Verweis auf die Autorisierung durch nationale Parlamente. In der Betrachtung des Krisenverlaufs fällt auf, dass im Gegensatz zu bisherigen Integrationsschritten eine eigenständig europäische Legitimationsquelle nicht oder nur sehr begrenzt herangezogen wurde.

\section{Die vertikale Zuständigkeitsverteilung: To bail out or not to bail out?}

Das Ebenendilemma stellte sich zunächst in der Frage, ob gemeinschaftliches Handeln als Antwort auf die Krise überhaupt wünschenswert sei. Die »Herren der Verträge« ${ }^{8}$ hatten in ihrer Betonung der Selbstverantwortung der Mitgliedstaaten für ihre finanzielle Stabilität eigentlich ein solches Handeln ausgeschlossen. Die sogenannte »No-BailoutKlausel«(Art. 125 TFEU) und das Verbot der direkten Staatsfinanzierung durch die EZB sollten den Gläubigern an den Finanzmärkten signalisieren, dass die Union (und ihre Mitglieder) nicht für etwaige finanzielle Probleme einzelner Mitgliedstaaten einstehen würde. Dieses Signal nahmen die Investoren offenbar nicht ernst. ${ }^{9}$ Die Risiken eines Kreditausfalls, ausgedrückt durch den Zinssatz staatlicher Anleihen, wurden über lange Zeiträume als zwischen den Euro-Mitgliedern weitgehend identisch bewertet.

Beim Eintritt des Extremfalls, der drohenden Zahlungsunfähigkeit Griechenlands im Frühjahr 2010, stellte sich daher die Frage, ob die Staats- und Regierungschefs an der ursprünglichen Konzeption festhalten sollten, oder ob die Investoren mit ihrer impliziten Annahme, die Union könne sich ein Beharren auf Prinzipien nicht leisten, recht behalten würden. Die Idee der Abschreckung, die die »No-Bailout« Klausel und das Verbot der direkten Staatsfinanzierung durch die EZB sowohl gegenüber den Regierungen als Schuldnern als auch den Finanzinvestoren als Gläubigern beinhalteten, erwies sich in jedem Fall als nicht geeignet, eine derartige Krise zu verhindern. Diese Entwicklung offenbart grundsätzliche Konstruktionsfehler des Vertrags von Maastricht.

Obwohl es in der europäischen Medienöffentlichkeit, nicht zuletzt der deutschen, im Verlauf der Krise in zunehmenden Maße Stimmen gab, die die Möglichkeit einer Rückkehr zu nationalen Währungen durchaus ernsthaft vertraten, stellten die zentralen politischen Akteure die Entscheidung für ein gemeinschaftliches Handeln zu keinem Zeitpunkt grundsätzlich in Frage. Dabei betonten die Entscheidungsträger neben den »sunk

8 Vgl. Bundesverfassungsgericht, Urteil vom 30. Juni 2009, 2 BvE 2/08, Randnote 231.

9 Vgl. Sachverständigenrat zur Begutachtung der gesamtwirtschaftlichen Entwicklung, Stabile Architektur für Europa - Handlungsbedarf im Inland, Jahresgutachten 2012/13, S. 102.

ZfP 60. Jg. 2/2013 
costs « der Währungsunion zuvorderst die desaströsen Folgen eines Auseinanderbrechens der Eurozone für das politische Fundamentalinteresse der europäischen Integration als Ganze: »Die gegenwärtige Krise des Euro ist die größte Bewährungsprobe, die Europa seit Jahrzehnten, ja wohl seit Unterzeichnung der Römischen Verträge im Jahre $1957 \mathrm{zu}$ bestehen hat. [...] Die Währungsunion ist eine Schicksalsgemeinschaft. Es geht deshalb um nicht mehr und nicht weniger als um die Bewahrung und Bewährung der europäischen Idee. ${ }^{10}$ Wie bereits in den Diskussionen zur Einführung einer gemeinsamen Währung zwischen Helmut Kohl und François Mitterrand wurden ökonomische Argumente von politischen Erwägungen überlagert, die unmittelbar und häufig indirekt die Bedeutung der Währungsunion als Eckpfeiler der Union für die (geo-) politische Verankerung der Mitgliedstaaten hervorhoben. ${ }^{11}$

Als Resultat der bisherigen Krisenmaßnahmen ist zu konstatieren, dass die Staats- und Regierungschefs der Mitgliedstaaten sich - erneut - für eine Stärkung der EU-Ebene als Antwort auf Defizite ihrer Problemlösungskapazität entschieden haben. Den klassischenen Theoremen des spill-over ${ }^{12}$ und der Hallstein'schen Sachlogik ${ }^{13}$ folgend haben die Staats- und Regierungschefs die Defizite vorangegangener Entscheidungen durch weitergehende Integrationsschritte überwinden müssen. In der vertikalen Beziehung zwischen Union und Mitgliedstaaten haben die Krisenreaktionen der Regierungen folglich - mehr als der Verfassungsvertrag und der Lissabonner Vertrag vorsahen - die Kompetenzen der EU Ebene ausgeweitet. Manche der Entscheidungen, insbesondere der Vertrag über die Stabilität, Koordinierung und Steuerung der Wirtschafts- und Währungsunion (der sogenannte »Fiskalpakt«), greifen in einem Maße in mitgliedstaatliche Hoheitsrechte ein, das als historische einschneidende Verlagerung von Zuständigkeiten bezeichnet werden kann. Die Budgethoheit nationaler Parlamente, bisher ein Grundstein demokratischer Legitimität, wird durch die neuen Regelungen in einem qualitativ deutlich höheren Maße eingeschränkt, als dies bisher durch den - in der Praxis nicht durchsetzungsfähigen - Stabilitäts- und Wachstumspakt der Fall war.

Gleichzeitig gab es im Verlauf der Krise vielerorts einflussreiche Stimmen, die die Grenzen einer weiterer Kompetenzverlagerungen betonten. Diese Stimmen fanden politisches Gehör. So wurden Maximalforderungen wie etwa nach einem direkten Eingriffsrecht der Kommission in nationale Budgetverfahren oder der Einführung gemeinsamer Schuldtitel nicht Folge geleistet. Dabei ist zu betonen, dass dieser »Souveränitätsreflex « nicht allein - und nicht einmal vorwiegend - von den Staats- und Regierungschefs

10 Angela Merkel, Regierungserklärung von Bundeskanzlerin Merkel zu den Euro-Stabilisierungsmaßnabmen, 19. Mai 2010.

11 Vgl. u.a. Hans-Peter Schwarz, Helmut Kobl. Eine Politische Biographie, München 2012, S. 397-439.

12 Ernst B. Haas, The Uniting of Europe. Political, Social and Economic Forces 1950-1957, London 1958. Vgl. auch Arne Niemann / Phillippe Schmitter, »Neofuntionalism« in: Antje Wiener / Thomas Dietz (Hg.), Theories of European Integration, Oxford 2009, S. 45-66.

13 Walter Hallstein, Der Unvollendete Bundesstaat. Europäische Erfabrungen und Erkenntisse, Düsseldorf/Wien 1969, S. $20 \mathrm{ff}$. Vgl. auch Wolfgang Wessels, »Walter Hallsteins integrationstheoretischer Beitrag: überholt oder vakant? « in: Wilfried Loth / William Wallace / Wolfgang Wessels (Hg.), Walter Hallstein: der vergessene Europäer?, Bonn 1995, S. 281-310. 
selber formuliert wurden. Neben der zumeist skeptischen Medienöffentlichkeit wiesen wiederholt nationale Verfassungsgerichte die Grenzen der Kompetenztransfers auf. So betonte das deutsche Bundesverfassungsgericht in seinem Urteil zur Griechenland-Hilfe und zum EFSF, der Deutsche Bundestag dürfe »seine Budgetverantwortung nicht durch unbestimmte haushaltspolitische Ermächtigungen auf andere Akteure übertragen. «14

\section{Agree to Disagree? Die Magnet- und Abstoßwirkungen eines Kerneuropas}

Eine wichtige Beobachtung bei den institutionellen Verschiebungen ist, dass die Entscheidung für die europäische Ebene oftmals nicht einhellig gefallen ist. Eine mögliche Lösung des gemeinsamen Ringens um eine Antwort auf das Ebenendilemma kann eben auch eine Einigung auf die Uneinigkeit sein. In der Tradition konstruktiver Enthaltungen, »opt-outs « und Ausnahmeregelungen haben auch die Krisenbewältigungsmaßnahmen gezeigt, dass nicht alle Mitgliedstaaten gewillt sind, die europäische Ebene als die adäquate Problemlösungsebene zu akzeptieren, ohne die Handlungen einer Mehrheit notwendigerweise zu blockieren. So betonte David Cameron in seiner lang erwarteten Rede zur Zukunft des Vereinigten Königreichs in der Europäischen Union die Notwendigkeit einer institutionellen Struktur, die die Verschiedenheit ihrer Mitglieder berücksichtige, von denen manche eine engere politische und wirtschaftliche Kooperation anstrebten, andere aber dieses Ziel »niemals « in Erwägung zögen. Nicht alle Mitgliedstaaten präferierten dasselbe Integrationsniveau, und die Gemeinschaft solle dies nicht erzwingen. ${ }^{15}$

In diesem Sinne wurden zwar Maßnahmen wie der »Fiskalpakt « oder der »Euro-Plus Pakt« von einer großen Mehrheit der EU-Mitgliedstaaten unterstützt, gleichzeitig aber von einer kleinen Kerngruppe »souveränistischer « Staaten, insbesondere Großbritannien, abgelehnt. Im Falle des Fiskalpakts verhinderte die fehlende Einstimmigkeit die Möglichkeit, diese Maßnahme direkt in EU-Recht aufzunehmen. Dies führte zu der rechtlich komplexen (und angefochtenen) Konstruktion eines völkerrechtlichen Vertrags jenseits des EU-Rahmens, der aber gleichzeitig direkt auf EU-Organe verweist und diese in seine Bestimmungen einbezieht. Eine weitere Möglichkeit einer solchen Flexibilisierung zeigt sich im Bezug auf die Diskussionen um eine Finanztransaktionssteuer, deren Verwirklichung von elf Mitgliedstaaten mithilfe des bisher selten genutzten Verfahrens der »verstärkten Zusammenarbeit« (Art. 20 EUV und Art. 326-334 AEUV) angestrebt wird, während sich die übrigen 16 daran nicht beteiligen wollen.

Doch nicht diese Maßnahmen allein sind Ausdruck der Uneinigkeit über die Problemlösungsebene. Bereits das Konstrukt der Eurozone an sich weist die Merkmale eines »Europa der mehreren Geschwindigkeiten« auf, wobei sich die Gruppe der »outs« in diesem Fall in einen kleineren Kern der Nicht-Willigen und eine größere Gruppe der (Noch-)Nicht-Könnenden teilt. Der anvisierte Beitritt Lettlands zur Eurozone weist

14 Bundesverfassungsgericht, Urteil vom vom 7. September 2011, 2 BvR 987/10, Leitsatz 3a.

15 David Cameron, Prime Minister David Cameron's speech on the future of the EU and the UK's relationship with it, 23. Januar 2013.

ZfP 60. Jg. 2/2013 
dabei auf eine trotz Krise nur wenig geminderte Attraktivität der europäischen Ebene in Fragen der Währungspolitik hin, obwohl sich gerade in Euro- (aber nicht unbedingt EU-) skeptischen Staaten wie Schweden die Ablehnung durch die Krise konsolidiert hat.

Als institutionellem Ausdruck dieser Entwicklungen ist auf den wachsenden Einfluss des Euro-Gipfels als politische Entscheidungsinstanz hinzuweisen - auch über seine 17 Mitglieder hinaus. Diese Institution der Staats- und Regierungschefs der Eurozone trat erstmals im Mai 2008 ad hoc als Reaktion auf die Auswirkungen der Finanzkrise zusammen, verstetigte sich jedoch im Zuge der dringlicher werdenden Probleme der Eurozone zusehends. Auf einem Treffen der Staats- und Regierungschefs am 26. Oktober 2011 einigten sich die Beteiligten schließlich auf eine Institutionalisierung dieses Gremiums, das nun mindestens zweimal im Jahr zusammentreten soll, »um strategische Orientierungen zu den Wirtschafts- und Haushaltspolitiken im Euro-Währungsgebiet vorzugeben «. ${ }^{16}$ Dabei wurden Bemühungen vorgenommen, die Arbeit des Euro-Gipfels so eng wie möglich mit denen des Europäischen Rats zu verzahnen. So sind sowohl Mitgliedschaft als auch administrative Strukturen des Euro-Gipfels denen des Europäischen Rats angepasst. Der Präsident der Kommission ist Mitglied, und die Wahl eines Präsidenten des Euro-Gipfels wird zeitgleich zur Wahl des Präsidenten des Europäischen Rats durchgeführt. ${ }^{17}$ Die Wahl Hermann Van Rompuys zum ersten Präsidenten des EuroGipfels unterstreicht diese Bemühungen. Nichtsdestoweniger entstand wiederholt der Eindruck, Entscheidungen des Euro-Gipfels erhöhten entweder die Exklusivität der Gruppe der Eurostaaten oder stellten den Europäischen Rat in wirtschaftspolitischen Fragen vor vollendete Tatsachen. Um diesen Befürchtungen entgegenzutreten sehen die nun veröffentlichten »Regeln für die Organisation der Arbeiten des Euro-Gipfels« vor, zumindest die nicht-Euro-Mitglieder des »Fiskalpakts « an Beratungen zu einer möglichen » ̈̈nderung der allgemeinen Architektur des Euroraums « zu beteiligen. ${ }^{18}$

In diesem Sinne geht von den Entscheidungen des Euro-Gipfels eine Magnetwirkung aus. Die verstärkte Nutzung der Formen differenzierter Integration hat nicht nur zu einer vertieften Spaltung innerhalb der EU geführt, sondern auch Bemühungen der »opt-out « Staaten beschleunigt, Möglichkeiten zu schaffen, eigene Interessen in die Verfahren des »Kerns « einzubringen. Nicht-Mitglieder der Eurozone - insbesondere Polen - erheben wiederholt und nachhaltig einen Anspruch, mit am Tisch der Entscheidungsträger zu sitzen.

Die ohnehin in Ansätzen bestehende Architektur eines »Europa der mehreren Geschwindigkeiten « gleicht im Zuge dieser Entwicklungen mehr und mehr der Konzeption eines »Europe á la carte «. ${ }^{19}$ Zudem scheinen viele der (im Sinne der Mitgliederzahl) »flexiblen « Maßnahmen, nicht zuletzt der Vorstoß zu einer Finanztransaktionssteuer, dem bisher ausschließlich Euro-Mitgliedstaaten zustimmen, auf einen konstanten Kern an

16 Erklärung des Euro-Gipfels, 8. November 2011, Randnote 31.

17 Vgl. Tobias Kunstein / Wolfgang Wessels, "Die Europäische Union in der Währungskrise: Eckdaten und Schlüsselentscheidungen « in: integration, Nr. 4 (2011), S. 308-322, hier S. 318.

18 Rat der Europäischen Union, Regeln für die Organisation der Arbeiten des Euro-Gipfels, 14. März 2013, Art. 4 Abs. 5.

19 Ralf Dahrendorf, A Third Europe? Third Jean Monnet Lecture, Florenz, 26. November 1979. 
»integrationswilligen « Mitgliedstaaten hinzuweisen. In diesem Sinne suggerieren die Entwicklungen eine Festigung »konzentrischer Kreise« (siehe Grafik 1).

Grafik 1: Konzentrische Kreise in der EU Wirtschafts- und Währungspolitik

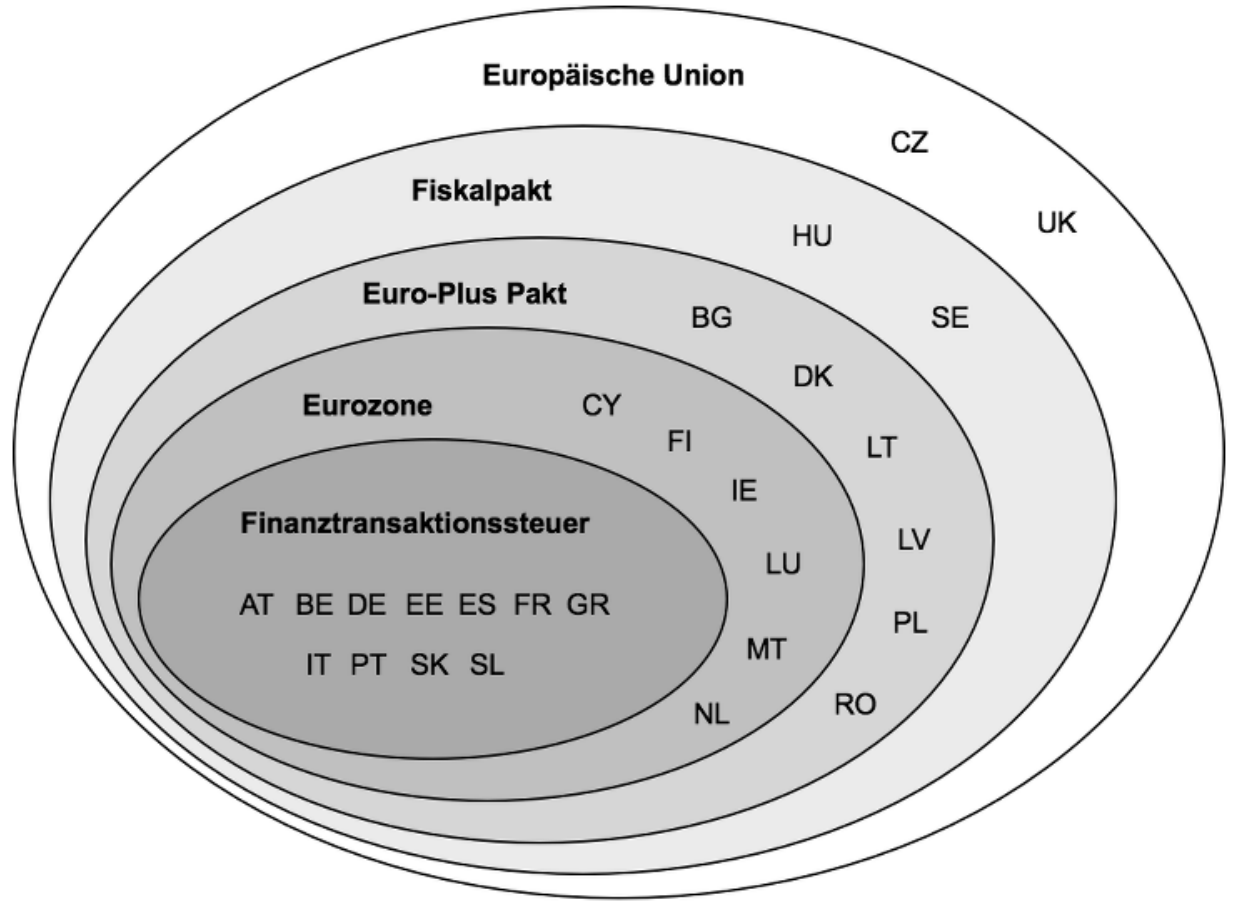

Quelle: Eigene Darstellung. ${ }^{20}$

Die horizontale Aufgabenverteilung: Wer soll der Hüter der Währungsunion sein?

Die Wahl der Europäischen Union als adäquate Problemlösungsebene bedingt aber keinesfalls im gleichen Zug eine Einigung auf horizontale Verfahren der Entscheidungsfindung, die klassisch als »supranational« bezeichnet werden. Vielmehr hat die Reaktion auf die Krise verdeutlicht, dass alle wichtigen Impulse von den Staats- und Regierungschefs im Europäischen Rat kommen. Der Kommission hingegen kann entgegen ihrem Anspruch als politischer »Agenda-Setzer « kaum ein eigenständiger Einfluss auf den Verlauf der Krisenreaktion attestiert werden. Ein wichtiges institutionelles Novum im Zuge der Krise ist die zentrale Rolle der Europäischen Zentralbank, die zunehmend Entscheidungen von hoher politischen Bedeutung trifft.

20 Angelehnt an Funda Tekin, Differentiated Integration at Work, Baden Baden 2012, S. 20.

ZfP 60. Jg. 2/2013 


\section{Der Europäische Rat als Schlüsselinstitution: Vorbehalt des nationalen Vetos}

Ohne Zweifel haben die politischen Führungspersonen den Europäischen Rat in seiner Rolle als zentraler Leitliniengeber und Entscheidungsorgan im Zuge der Krise gefestigt und ausgebaut. Die herausgehobene Rolle des Europäischen Rats - und zunehmend des Euro-Gipfels - als zentraler Krisenmanager zeigt sich bereits in der Häufigkeit seiner Treffen über den Verlauf der Krise. Legte der Vertrag von Lissabon noch zwei reguläre Treffen pro Halbjahr fest, häuften sich nach dem Eintritt der »Schuldenkrise« die außerplanmäßigen Zusammenkünfte. Von 2010 bis 2012 trat der Europäische Rat in einer Mischung aus regulären Tagungen, außergewöhnlichen Sitzungen und informellen Treffen der Staats- und Regierungschefs insgesamt 19 Mal zusammen. Hinzu kommen acht nun als Euro-Gipfel institutionalisierte Treffen der Staats- und Regierungschefs der Eurozone. Alle zentralen Maßnahmen zur Krisenbewältigung fanden Ihren Ursprung in Deliberationen dieser Institutionen. Dabei gingen die Staats- und Regierungschefs in den Details ihrer Vorgaben weit über eine bloße Rolle als Impulsgeber hinaus. In vertraglich in dieser Form nicht festgelegter Weise bestimmten Euro-Gipfel und Europäischer Rat seit dem ersten informellen Treffen am 11. Februar 2010, das sich mit der eskalierenden Haushaltssituation in Griechenland beschäftigte, alle zentralen Einzelheiten der zu treffenden Maßnahmen (siehe Übersicht 2).

Übersicht 2: Maßnahmen des Europäischen Rats und des Euro-Gipfels

\begin{tabular}{|l|l|}
\hline 11. Februar 2010 & $\begin{array}{l}\text { Informelles Treffen der EU Staats- und Regierungschefs. Ers- } \\
\text { te Stellungnahme zur Budgetsituation in Griechenland. }\end{array}$ \\
\hline 25.-26. März 2010 & $\begin{array}{l}\text { Treffen der Staats- und Regierungschefs der Eurozone und } \\
\text { Europäischer Rat. Bilaterale Darlehen an die griechische Re- } \\
\text { gierung und Einsetzung der »Arbeitsgruppe wirtschaftspoli- } \\
\text { tische Steuerung (Van Rompuy Taskforce). }\end{array}$ \\
\hline 7.-8. Mai 2010 & $\begin{array}{l}\text { Treffen der Staats- und Regierungschefs der Eurozone. Eini- } \\
\text { gung auf Prinzipien der»Europäischen Finanzstabilisierungs- } \\
\text { faszilität« (EFSF) und des } \\
\text { rungsmechanismus « (EFSM). }\end{array}$ \\
\hline 17. Juni 2010 & $\begin{array}{l}\text { Europäischen Finanzstabilisie- } \\
\text { gung und Eintelligentes, nachhaltiges und integratives Wachs- } \\
\text { tum« (Europa 2020), sowie neue Leitlinien für haushaltspoli- } \\
\text { tische Überwachung und wirtschaftspolitische Koordination. } \\
\text { Prinzipien für ein »Europäisches Semester«. }\end{array}$ \\
\hline 28.-29. Oktober 2010 & $\begin{array}{l}\text { Europäischer Rat. Annahme des Berichts der »Arbeitsgruppe } \\
\text { wirtschaftspolitische Steuerung« (Van Rompuy Taskforce). }\end{array}$ \\
\hline $\begin{array}{l}\text { 16.-17. Dezember } \\
\text { 2010 }\end{array}$ & $\begin{array}{l}\text { Europäischer Rat. Beschluss einer Vertragsänderung zur Ein- } \\
\text { richtung des (permanenten)»Europäischen Stabilitätsmecha- } \\
\text { nismus« (ESM). }\end{array}$ \\
\hline
\end{tabular}




\begin{tabular}{|l|l|}
\hline 11. März 2011 & $\begin{array}{l}\text { Treffen der Staats- und Regierungschefs der Eurozone. Eini- } \\
\text { gung auf einen } \\
\text { geren wirtschaftspolitischen Koordinierung. }\end{array}$ \\
\hline 21. Juli 2011 & $\begin{array}{l}\text { Treffen der Staats- und Regierungschefs der Eurozone. Eini- } \\
\text { gung auf ein weiteres Hilfspaket für Griechenland und Er- } \\
\text { weiterung des EFSF. }\end{array}$ \\
\hline 8.-9. Dezember 2011 & $\begin{array}{l}\text { Europäischer Rat und Euro-Gipfel. Einigung auf einen } \text { Ner- } \\
\text { trag über Stabilität, Koordinierung und Steuerung in der Wirt- } \\
\text { schafts- und Währungsunion« (Fiskalpakt). }\end{array}$ \\
\hline 28.-29. Juni 2012 & $\begin{array}{l}\text { Europäischer Rat und Euro-Gipfel. Einigung auf einen »Pakt } \\
\text { für Wachstum und Beschäftigung«. }\end{array}$ \\
\hline 13. Dezember 2012 & $\begin{array}{l}\text { Europäischer Rat. Einigung auf Grundzüge einer gemeinsa- } \\
\text { men Bankenaufsicht. }\end{array}$ \\
\hline
\end{tabular}

Quelle: Eigene Darstellung. ${ }^{21}$

Aber auch jenseits dieser richtungsweisenden Entscheidungen hat sich der Europäische Rat dauerhaft - und mehr als zuvor - in Prozesse der Politikherstellung innerhalb der Wirtschafts- und Währungsunion »hineingeschrieben«. So ist der Europäische Rat zentral in die jährliche Beschlussfassung zu Maßnahmen im Rahmen des neu geschaffenen »Europäischen Semesters « und des »Euro-Plus Pakts « eingebunden. Im Kontext der nun stärker zu koordinierenden Wirtschafts- und Haushaltspolitiken der EU-Mitgliedstaaten und der Umsetzung der Strategie »Europa 2020« legt er zentrale wirtschafts- und haushaltspolitische Prioritäten der EU fest und billigt länderspezifische Empfehlungen der Kommission.

Die Krisenbewältigung hat auch das erst 2009 mit dem Lissabonner Vertrag eingeführte Amt des Vollzeitpräsidenten des Europäischen Rats geprägt. Nach seinem eigenen Rollenverständnis hat er seine - auch vertraglich vorgegebene - Aufgabe (Art. 15 Abs. 6 EUV) als »Facilitator « und »Konsensmakler « intensiv und - nach dem Bericht von Beteiligten - auch erfolgreich wahrgenommen. Eine charismatische Führungsrolle als Sprecher der Union hat und konnte er nicht anstreben. Die Mitglieder des Europäischen Rats haben ihn mit dem Entwurf von Berichten und Positionen beauftragt, die vor der Einrichtung dieses Amts weitgehend vom Präsidenten der Kommission ausgeübt wurden. Es ist zu erwarten, dass die Rollenwahrnehmung des ersten Amtsinhabers das Rollenverständnis auch in Zukunft prägen wird. ${ }^{22}$

Mit der Bedeutungszunahme des Europäischen Rats sind auch Verschiebungen der Machtverhältnisse zwischen den Mitgliedstaaten im Europäischen Rat zu konstatieren. Entgegen ursprünglichen Erwartungen, dass in einer größeren Union das deutsch- fran-

21 Angelehnt an Kunstein / Wessels, Die Europäische Union in der Währungskrise, aaO. (FN 17), S. 309-310.

22 Vgl. Wolfgang Wessels, European Council, Basingstoke, im Erscheinen; Wolfgang Wessels / Thomas Traguth »Der hauptamtliche Präsident des Europäischen Rates:,Herr' oder,Diener' im Haus Europa? in: integration, Nr. 4 (2011), S. 297-311.

ZfP 60. Jg. 2/2013 
zösische Tandem an Einfluss verlieren würde, ${ }^{23}$ übernahmen Nicolas Sarkozy und Angela Merkel bei allen entscheidenden Vorhaben eine zentrale Führungsrolle. Paradigmatisch für diese bilateralen Vorstöße war das Treffen der beiden Regierungschefs im französischen Deauville im Herbst 2010, bei dem sich die französische und deutsche Seite im Vorfeld des Europäischen Ratstreffens auf zentrale Parameter der Reform des Stabilitätsund Wachstumspakts einigten. Dieses Vorgehen stieß auf vehemente Kritik der anderen Partner, die sich in der Entscheidungsfindung vernachlässigt sahen. ${ }^{24}$

Seit der Wahlniederlage Sarkozys im Frühjahr 2012 hat sich eine ähnliche Partnerschaft zwischen Angela Merkel und dem sozialistischen Präsidenten Hollande noch nicht im selben Maße etabliert. Gleichzeitig ist die zentrale Position der deutschen Bundesregierung in der Debatte um eine adäquate Krisenlösung, die nicht zuletzt aus ihrer ökonomischen Stärke resultiert, zunehmender Kritik ausgesetzt. Mehr und mehr wird die deutsche Bundesregierung als - wenn auch widerwilliger - europäischer Hegemon gesehen, der seine Forderung nach wirtschafts- und haushaltspolitischer »Austerität « weitgehend gegen den Willen der Partner durchsetzt. ${ }^{25}$ Dabei sieht sich die deutsche Bundesregierung vor dem Dilemma, gleichzeitig einer drohenden außenpolitischen Isolation entgehen wirken zu müssen sowie nach innen - zumal in einem Wahljahr - Krisenbewältigungsmaßnahmen rechtfertigen zu müssen, die in Deutschland sowohl in der Öffentlichkeit als auch in den Regierungsparteien selbst äußerst umstrittenen sind.

Die Verhandlungsmacht der »Empfängerländer « dagegen hat sich in der Krise auf ein Minimum reduziert. Ihr wichtigstes Druckmittel war im Wesentlichen die Möglichkeit der (finanziellen) Selbstzerstörung, die mit einiger Wahrscheinlichkeit die Währungsunion als Ganzes bedroht hätte. Dabei schien diese Option in der Abwägung des geringeren Übels in mehreren Situationen, insbesondere im Fall von Griechenland und $\mathrm{Zy}$ pern, aus der Sicht mancher Betroffener gar nicht so abwegig.

In der Gesamtbetrachtung der Rolle des Europäischen Rats im Verlauf der Krise setzt sich das vermeintliche Paradox fort, dass sich dieses intergouvernmentale Organ »par excellence« verantwortlich für Vertiefungsschritte historischen Ausmaßes zeigt. In der Breite seiner Entscheidungen entwickelt der Europäische Rat die Schlüsselelemente einer "gouvernement économique «. ${ }^{26}$ In diesem Vorgehen verdeutlicht sich das Bestreben zumindest maßgeblicher - Staats- und Regierungschefs, zentrale Maßnahmen insbeson-

23 Vgl. Joachim Schild, »Mission Impossible? The Potential for Franco-German Leadership in the Enlarged EU« in: Journal of Common Market Studies 48, Nr. 5 (2010), S. 1367-1390; William E. Paterson, »Did France and Germany Lead Europe? A Retrospect« in: Jack Hayward (Hg.), Leaderless Europe, Oxford/New York 2008, S. 89-112.

24 Vgl. u.a. Werner Mussler, »Stabilitätspakt á la Deauville« in: Frankfurter Allgemeine Zeitung vom 20. Oktober 2011, S. 11.

25 Vgl. »Don't make us Führer« The Economist, 13. April 2013; William E. Paterson, »The Reluctant Hegemon? Germany Moves Centre Stage in the European Union» in: Journal of Common Market Studies 49, Annual Review (2011), S. 57-75; Wolfgang Wessels, European Council, aaO. (FN 22).

26 Vgl. Commissariat Général du Plan, Le gouvernement économique de la zone euro; rapport du groupe présidé par Robert Boyer, Paris 1999; Wolfgang Wessels, European Council, aaO. (FN 22). 
dere im Hinblick auf eine strengere Überwachung nationaler Wirtschafts- und Haushaltspolitik unter der Vorgabe der Einstimmigkeit auszuhandeln. Selbst wenn der Problemlösungsinstinkt die Wahl der europäischen Ebene als Lösungsinstanz favorisiert, zeigen diese Krisenreaktionen deutlicher als zuvor einen starken Letztentscheidungsvorbehalt der Mitgliedstaaten. Der Europäische Rat hat sich damit als Schlüsselorgan für einen Fusionsprozess mehrerer Handlungsebenen und Instrumente erwiesen. ${ }^{27}$

\section{Die Kommission: Klarer Verlierer oder stiller Gewinner?}

Der bestehende Trend zum Einflussverlust der Kommission als »Agenda-Setzer « gegenüber dem Europäischen Rat setzt sich auch im Verlauf der Krise fort. Eine Vielzahl ihrer Initiativen fanden bei den Staats- und Regierungschefs kein oder nur indirekt über die Vorschläge der »Van Rompuy Taskforce« Gehör.

Zunächst ist hervorzuheben, dass die Kommission sich von Beginn der Krisenerscheinungen an aktiv an den Diskussionen um mögliche Lösungsansätze beteiligte und wiederholt versuchte, neue Impulse in die Auseinandersetzungen einfließen zu lassen. Dabei scheuten insbesondere Kommissionspräsident Barroso und Währungskommissar Rehn nicht den Konflikt mit den Mitgliedstaaten und stellten in offenen Briefen und Medienbeiträgen wiederholt die bis dahin gefundenen Maßnahmen der Regierungen in Frage. ${ }^{28}$ Gleichzeitig legte die Kommission regelmäßig eigene Vorschläge vor, die in ihrer anvisierten Verlagerung nationalstaatlicher Kompetenzen auf die EU-Ebene teilweise erheblich über die Kompromisse in Europäischem Rat und Euro-Gipfel hinausgingen. Diese Strategie erwies sich jedoch bisher als nur begrenzt erfolgreich.

Repräsentativ für dieses Vorgehen waren die Überlegungen der Kommission zur Einrichtung von gemeinsamen Staatsanleihen (im allgemeinen Sprachgebrauch vorwiegend "Eurobonds « genannt, von der Kommission aber vorsichtig "Stabilitätsanleihen « getauft)..$^{29}$ Derartige gemeinschaftliche Anleihen waren auch zuletzt noch Teil des von der Kommission im November 2012 vorgestellten Konzepts »für eine vertiefte und echte Wirtschafts- und Währungsunion «, das den Beitrag der Kommission zu dem vom Europäischen Rat in Auftrag gegebenen »Berichts der vier Präsidenten « darstellte und das der Europäische Rat im Dezember 2012 »zur Kenntnis nahm «.30 Obwohl die neu gewählte französische Regierung unter Präsident Hollande das Prinzip solcher Anleihen durchaus wohlwollend aufnahm, sind die Pläne der Kommission aufgrund der sonst weit verbreiteten Skepsis - nicht zuletzt in Deutschland - gegenüber weiter reichenden finanziellen Verpflichtungen bisher von den Staats- und Regierungschefs nicht ernsthaft

27 Vgl. Wolfgang Wessels, European Council, aaO. (FN 22); Wolfgang Wessels, »The European Council. Beyond the Traditional View towards a Fusion « in Jonathan Gérard Cohen (Hg.): Chemins d'Europe. Mélanges en l'honneur de Jean Paul Jacqué, Paris 2010, S. 751-764.

28 Vgl. Udo Diedrichs, »Europäische Kommission« in: Werner Weidenfeld / Wolfgang Wessels (Hg.), Jabrbuch der Europäischen Integration 2011, Baden-Baden 2011, S. 81-90, hier S. 81.

29 Europäische Kommission, Grünbuch über die Durchfübrbarkeit der Einführung von Stabilitätsanleiben (COM(2011) 818), 23. November 2011.

30 Europäischer Rat, Schlussfolgerungen, 14. Dezember 2012, Randnote 3.

ZfP 60. Jg. 2/2013 
weiter verfolgt worden. ${ }^{31}$ Ein ähnliches Schicksal widerfuhr dem Vorschlag der Kommission zu einem separaten Budget der Eurozone ${ }^{32}$ ebenso wie ihrem frühen Vorstoß, den »einheitlichen Aufsichtsmechanismus « für systemrelevante Banken bei der Europäischen Bankenaufsichtsbehörde in London anzusiedeln ${ }^{33}$ - und nicht bei der EZB, wie der Europäische Rat schließlich beschloss.

Erfolgreicher war die Kommission mit dem als »Sixpack « bezeichneten Maßnahmenpaket zur Stärkung der wirtschaftspolitischen Koordination und haushaltspolitischen Überwachung der Mitgliedstaaten, das die Kommission in einer Art » Wettlauf ${ }^{34}$ mit der Arbeit der »Van Rompuy Taskforce« im September 2010 vorlegte. Der Inhalt des »Sixpack «nimmt Teile des Abschlussberichts der »Van Rompuy Taskforce« vorweg und ist daher nicht unbedingt als eigenständiger Beitrag der Kommission zu werten. Mehr eigenständige Initiative ist in den Vorschlägen der Kommission vom November 2011 zur Stärkung ihrer Position in der Überwachung nationaler Haushalte im Rahmen des Stabilitäts- und Wachstumspakts und des »Europäischen Semesters « zu finden (»TwoPack «), über die nun zwischen Rat und Europäischem Parlament Einigung besteht.

Die untergeordnete Rolle der Kommission bei der politischen Gestaltung der neuen wirtschafts- und haushaltspolitischen Vorgaben für die Mitgliedstaaten darf nicht darüber hinwegtäuschen, dass die Staats- und Regierungschefs der Kommission bei der Umsetzung nahezu aller Krisenlösungsmechanismen zentrale Aufgaben der Überwachung nationaler Wirtschafts- und Finanzpolitik übertragen haben. Die bestehende Rolle der Kommission als Hüter von eingegangenen Verpflichtungen ist daher durchaus gestärkt. ${ }^{35}$

Die Kompetenzen der Kommission als »Watchdog «im Rahmen des Stabilitäts- und Wachstumspakts wurden durch die Maßnahmen des »Sixpack « deutlich ausgebaut. Zentral ist dabei die Umkehrung der Mehrheitsanforderung im Rat bei der Einleitung des »korrektiven Arms « des Pakts. Die Vorgaben der Kommission im Rahmen des »Verfahrens wegen übermäßigem Defizit « haben in Zukunft automatisch Bestand, wenn nicht eine qualifizierte Mehrheit im Rat die Kommission überstimmt. Die Umsetzung der Kommissionsvorgaben in einem solchen Fall - bis hin zu Sanktionen - wird dadurch wahrscheinlicher. Des weiteren haben der Rat und das Europäisches Parlament die Überwachungskompetenzen der Kommission von nationalen Staatshaushalten auf weitergehende Fragen der wirtschaftlichen Steuerung ausgedehnt. Im neu geschaffenen »Verfahren bei einem (übermäßigen) makroökonomischen Ungleichgewicht« ist die Kommission in der Lage, analog zum Defizitverfahren - letztendlich sanktionsbewährte

31 Vgl. Udo Diedrichs, »Europäische Kommission « in: Werner Weidenfeld / Wolfgang Wessels (Hg.), Jabrbuch der Europäischen Integration 2012, Baden-Baden 2012, S. 91-100, hier S. 93.

32 Europäische Komission, Ein Konzept für eine vertiefte und echte Wirtschafts- und Währungsunion. Auftakt für eine europäische Diskussion (COM(2012) 777), 28. November 2012, S. $36 \mathrm{ff}$.

33 Vgl. Eilis Ferran / Valia Babis, The European Single Supervisory Mechanism, University of Cambridge Faculty of Law Research Paper Nr. 10 (2013), S. 2.

34 Daniela Schwarzer, »Economic Governance in der Eurozone« in: Aus Politik und Zeitgeschichte, Nr. 4 (2012), S. 17-24, hier S. 19.

$35 \mathrm{Vgl}$. Andrew Moravcsik, The Choice for Europe. Social Purpose and State Power from Messina to Maastricht, Ithaca, NY 1998, S. 9. 
- wirtschaftspolitische Empfehlungen an betroffene Staaten zu richten, die Bestand haben, wenn sich nicht eine qualifizierten Mehrheit des Rats dagegen ausspricht. Zum Zweck der Kontrolle kann die Kommission zudem »Missionen zur verstärkten Überwachung « in den betroffenen Mitgliedstaaten durchführen. ${ }^{36}$

Die Maßnahmen des »Two-Packs« schreiben die Stärkung der Kommission in der Kontrolle nationaler Budgets fort. Die Mitgliedstaaten der Eurozone verpflichten sich nun, einen Entwurf ihres jährlichen Haushaltsplans vor der parlamentarischen Verabschiedung der Kommission vorzulegen. Die Kommission prüft, ob diese Haushalte mit den Vorgaben des Stabilitäts- und Wachstumspakts und den Empfehlungen aus dem Europäischen Semester übereinstimmen und kann bei Abweichungen eigene Standpunkte formulieren, die sie der Euro-Gruppe vorlegt. Besonders gestärkte Kompetenzen der Kommission sieht das »Two-Pack « gegenüber Staaten der Eurozone vor, die sich in einem »Verfahren wegen übermäßigem Defizit« befinden - 2012 immerhin 13 Mitglieder der Eurozone ${ }^{37}$ - oder europäische Finanzhilfen beanspruchen. Solche Staaten werden einer besonderen Haushaltskontrolle ausgesetzt, die auch regelmäßige Überwachungsmissionen der Kommission beinhalten kann. Insgesamt kann in diesem Teil der Verordnungen des »Two-Packs« eine Konsolidierung des Ansatzes der »Task Force für Griechenland» erkannt werden, die die Kommission im Juli 2011 zur stärkeren Überwachung der griechischen Reformbestrebungen eingerichtet hatte. ${ }^{38}$

Das Ausbleiben eines eigenständigen Einflusses auf die Konzipierung der Krisenreaktionen heißt demnach nicht, dass nicht auch die Kommission "gestärkt« aus der Krise hervorgehen könnte. Während der Anspruch der Kommission, als alleinige europäische Wirtschaftsregierung angesehen zu werden, ${ }^{39}$ sich im Zuge der Krise nicht verwirklicht hat, kann sie doch mehr und mehr als »ausführender Arm« des Leitlinien setzenden Europäischen Rats in der Wirtschafts- und Fiskalpolitik gesehen werden. Die Reichweite ihrer Kompetenzen hat der Europäischen Rat im Hinblick auf makro-ökonomische Supervision bedeutend ausgeweitet. In diesem Vorgehen lässt sich ein Kompromiss der Staats- und Regierungschefs im Hinblick auf das Entscheidungsdilemma feststellen. Während sie in der Konzeption der Krisenbewältigung auf einem Letztentscheidungsvorbehalt beharren, setzt sich eine Suche nach Effizienz in der Umsetzung der gefundenen Lösung durch.

36 Verordnung Nr. 1176/2011 des Europäischen Parlaments und des Rates vom 16. November 2011 über die Vermeidung und Korrektur makroökonomischer Ungleichgewichte, Art. 9 Abs. 3.

37 Europäische Kommission, »European Report on Public Finances in EMU, European Economy 4/2012, Juli 2012.

38 Vgl. Europäische Kommission, Fragen und Antworten zur Task Force für Griechenland (Memo/11/599), 13. September 2011.

39 »Indeed, within the Community competences, the Commission is the economic government of the Union, we certainly do not need more institutions for this. « José Manuel Barroso, European renewal - State of the Union Address 2011, Rede vor dem Europäischen Parlament in Strasbourg am 28. September 2011. 


\section{Die EZB: "Expertokratie« an Stelle politischer Entscheidungen?}

Bisher weitgehend im toten Winkel politikwissenschaftlicher Betrachtung hat sich die Europäische Zentralbank als zentrales Organ zur Überwindung der drastischsten Krisensymptome erwiesen. Letztendlich trauten die Finanzinvestoren lediglich der EZB zu, über ausreichend Willen und Kapazitäten zur Sicherung der finanziellen Stabilität zentraler Mitglieder der Eurozone wie Spanien oder Italien zu verfügen. Dies erklärt auch die relativ verhaltene Reaktion der »Finanzmärkte « auf die chaotisch verlaufene Einigung auf ein Hilfspaket für Zypern im März 2013. Solange das Versprechen der Zentralbank, »alles « zu tun, was zur Rettung der Währungsunion notwendig erscheint ${ }^{40}$ Bestand hat - und von den Investoren für glaubwürdig gehalten wird - scheint selbst größte Uneinigkeit der Staats- und Regierungschefs nicht zu derselben Marktvolatilität zu führen, die noch Anfang 2012 das Resultat solcher Ereignisse gewesen wäre.

In dieser Entwicklung führt die mit dem Vertrag von Maastricht getroffene Wahl der Staats- und Regierungschefs zur Delegation weitgehender geldpolitischer Kompetenzen an eine unabhängige Europäische Zentralbank zu Konsequenzen, die die ursprünglichen Entscheidungsträgern wohl weder geplant noch vorhergesehen hatten. Insbesondere Vertreter des deutschen Ordo-Liberalismus, nach dessen Vorstellungen die EZB entworfen wurde, erkennen in den Aktionen der EZB ihre ursprüngliche Konzeption nicht wieder. ${ }^{41}$ Der Konflikt über die geldpolitische Ausrichtung lässt sich am Abstimmungsverhalten im EZB-Rat ablesen. Die deutschen Vertreter wurden in der Entscheidungsfindung über das Vorgehen der EZB wiederholt überstimmt. ${ }^{42}$ Diese Entwicklung veranlasste letztlich den Rücktritt sowohl des Präsidenten der deutschen Bundesbank Axel Weber am 11. Februar 2011 als auch des EZB Direktoriumsmitglieds Jürgen Stark am 9. September $2011 .^{43}$

In der Tat legte die EZB im Zuge der Krise ihr Mandat zur Gewährleistung der Preisstabilität (Art. 127 AEUV) zunehmen expansiv aus. In einer ersten Reaktion auf die sich abzeichnende Staatsschuldenkrise begann die EZB im Mai 2010 damit, Staatsanleihen der betroffenen Staaten auf dem Sekundärmarkt (also von Finanzinstituten) zu kaufen, um einer drohende »Illiquiditätsspirale« auf dem Markt für staatliche Anleihen Einhalt zu

40 » Within our mandate, the ECB is ready to do whatever it takes to preserve the euro. And believe me, it will be enough. « Rede Mario Draghis, Präsident der Europäischen Zentralbank auf der »Global Investment Conference« in London, 26. Juli 2012.

41 Vgl. u.a. Jenaer Allianz, »Wirtschaftsverfassung statt Wirtschaftsregierung. Frankfurter Aufruf für eine ordnungspolitische Weichenstellung in Europa« in: Frankfurter Allgemeine Zeitung vom 22. Juni 2012, S. 12; Jürgen Stark, »Die EZB verliert ihre Unabhängigkeit « in: Handelsblatt vom 28. August 2012.

42 Vgl. u.a. Jörg Krämer, »Mehr Stimmen für die Bundesbank« in: Frankfurter Allgemeine Zeitung vom 26. März 2012, S. 20; Christian Siedenbiedel, »Mario Draghis Notenbank in: Frankfurter Allgemeine Sonntagszeitung vom 16. Oktober 2011, S. 38.

43 Siehe Kunstein / Wessels, Die Europäische Union in der Währungskrise, aaO. (FN 17), 315; Martin Selmayr, »Europäische Zentralbank « in: Werner Weidenfeld / Wolfgang Wessels (Hg.), Jabrbuch der Europäischen Integration 2012, Baden-Baden 2012, S. 111-124, hier S. 112. 
gebieten. ${ }^{44}$ Kritiker sahen in diesem Vorgehen einen Verstoß gegen das in Artikel 123 AEUV festgelegte Verbot der direkten Finanzierung von Staatsschulden durch die EZB. ${ }^{45}$ Gleichwohl blieb ein derartiger Ankauf von Schuldtiteln ein zentrales Instrument der Krisenbewältigung. Nicht zuletzt wurde Mario Draghis oben zitiertes Versprechen zur Eurorettung zwei Monate später von der Ankündigung begleitet, die Anleihenankäufe ohne konkret begrenzten Umfang fortzuführen. ${ }^{46}$ Im Dezember 2011 beschloss die EZB zudem, europäischen Finanzinstituten umfangreiche Finanzmittel zu sehr günstigen langfristigen Konditionen zur Verfügung zu stellen. Dieses Vorgehen wurde allgemein als Reaktion auf die wachsende Zurückhaltung europäischer Banken gesehen, Kredite an Unternehmen zu vergeben. Dabei ist die Versorgung der Realwirtschaft mit ausreichend Investitionskapital nicht im Mandat der EZB enthalten. Die Zentralbank rechtfertigt ihr Vorgehen mit der Notwendigkeit der Sicherung zentraler Transmissionsriemen zwischen ihren geldpolitischen Entscheidungen und den Markteffekten. In einem durch die Krisenerscheinungen instabil gewordenen Markt könne die EZB ihr Mandat zur Sicherung der Preisstabilität nicht wahrnehmen. Der Erhalt und die Stabilisierung des Eurosystems sei daher essentielle Vorbedingung zur Erfüllung ihres Mandats. $^{47}$

Ein potenziell noch bedeutenderer Wandel der institutionellen Position der EZB ist in ihrer zunehmenden Rolle als Politikgestalter zu finden, die ebenso kritisch hinterfragt wird. ${ }^{48}$ So war eine Delegation der Europäischen Zentralbank - zusammen mit Vertretern der Kommission und des Internationalen Währungsfonds - teil der Gruppe, im weiteren Verlauf der Krise »Troika« getauft, die im April und Mai 2010 in Griechenland die Vorgaben für ein finanzielles Rettungspaket erarbeitete und die auch weiterhin deren Einhaltung kontrolliert. Diese Funktion nahm die EZB auch im Kontext der darauffolgenden Finanzhilfen an Portugal, Irland und nun auch Zypern ein. Fernab der Festlegung geldpolitischer Leitimpulse ist die Europäische Zentralbank auf diese Weise zentral in die Formulierung und Kontrolle wirtschafts- und finanzpolitischer Vorgaben - etwa Reformen des Rentensystems oder der Flexibilisierung des Arbeitsmarkts - an die Empfängerstaaten involviert. Analog dazu war nicht zuletzt die konkrete Drohung der EZB, in der sich zuspitzenden finanziellen Notsituation die zyprische Zentralbank nicht mehr mit Finanzmitteln zu versorgen ${ }^{49}$ ausschlaggebend für die Entscheidung der zyprischen

44 Vgl. Francesco Drudi / Alain Durré / Francesco Paolo Mongelli, »The Interplay of Economic Reforms and Monetary Policy: The Case of the Eurozone« in: Journal of Common Market Studies 50, Nr. 6 (2012), S. 881-898, hier S. 889.

45 Vgl. u.a. Rainer Wernsmann, »Nicht über dem Recht« in: Frankfurter Allgemeine Zeitung vom 20. September 2012, S. 8.

$46 »$ No ex ante quantitative limits are set on the size of Outright Monetary Transactions. « Europäische Zentralbank, Technical features of Outright Monetary Transactions, Pressmitteilung vom 6. September 2012.

47 Vgl. Drudi / Durré / Mongelli, The Interplay of Economic Reforms and Monetary Policy, aaO. (FN 44), S. 390.

48 Vgl. u.a. Patrick Welter, »Die Fehlkonstruktion der Troika« in: Frankfurter Allgemeine Zeitung vom 4. April 2013, S. 9.

49 Europäische Zentralbank, Governing Council decision on Emergency Liquidity Assistance requested by the Central Bank of Cyprus, Pressemitteilung vom 21. März 2013.

ZfP 60. Jg. 2/2013 
Regierung im März 2013, die Bedingungen der Euro-Gruppe für ein Rettungspaket anzunehmen und auf eine Belastung versicherter Bankeinlagen zu verzichten.

Auch auf die politische (Neu-)Gestaltung der Wirtschafts- und Währungsunion hat die Europäische Zentralbank mit dem Ausbruch der Krise erstmals direkt Einfluss genommen. ${ }^{50}$ Neben ihren diversen Positionspapieren und öffentlichen Stellungnahmen zu möglichen Krisenreaktionsmaßnahmen - so etwa zum Anlass der Verleihung des Aachener Karlspreises an Präsident Trichet ${ }^{51}$ - ist eine neue Rolle des EZB-Präsidenten als "Stichwortgeber" im Europäischen Rat erkennbar. ${ }^{52}$ In diesem Sinne leitete JeanClaude Trichets energische Darstellung der Ernsthaftigkeit der finanziellen Situation im Euroraum eine Einigung des Europäischen Rats auf den ersten finanziellen Rettungsschirm im Mai 2010 ein. ${ }^{53}$ Ebenso verweisen Kommentare auf die zentrale Rolle des neuen EZB-Präsidenten Mario Draghi in den Beratungen des Europäischen Rats im Dezember 2011 zur Einrichtung des »Fiskalpakts « sowie der Einigung auf einen »Pakt für Wachstum und Beschäftigung « im Juni 2012.54

In der Gesamtsicht wird deutlich, dass sich die Europäische Zentralbank in keiner Weise als kontrollunterworfener $» A g e n t{ }^{55}$ der Mitgliedstaaten zur Durchführung einer gemeinsamen Geldpolitik versteht, sondern sich als unabhängiger »Treuhänder «56 zentral mit der Aufrechterhaltung und Stabilisierung des Euro-Systems vertraut sieht - und zu diesem Ziel jedes ihr zur Verfügung stehende Mittel aufwendet. Dass dieses Auftreten zu einer weitgehenden Beruhigung der Krisenentwicklungen geführt hat, lässt sich als Erfolg der Effizienzsuche der Mitgliedstaaten bereits in Maastricht interpretieren. Gleichzeitig birgt die (Neu-)Interpretation des Mandats der EZB als allgemeines Mandat zur Euro-Rettung die Gefahr eines Kontrollverlusts der Mitgliedstaaten. Politische Entscheidungsträger werden zunehmend von nur indirekt legitimierten Experten vor vollendete Tatsachen gesetzt - letztendlich aber haften die Regierungen der Mitgliedstaaten für eventuelle Verluste der Zentralbank.

\section{Auf der Suche nach Legitimität: Die Rückkehr der nationale Ebene}

Eine deutliche Entscheidung haben die Staats- und Regierungschefs im Hinblick auf das Legitimationsdilemma getroffen. Die neuen Mechanismen werden ausschließlich über

50 Vgl. Martin Selmayr, »Europäische Zentralbank «in: Werner Weidenfeld / Wolfgang Wessels (Hg.), Jahrbuch der Europäischen Integration 2010, Baden-Baden 2010, S. 95-106, hier S. 106.

51 Vgl. Martin Selmayr, »Europäische Zentralbank « in: Werner Weidenfeld / Wolfgang Wessels (Hg.), Jahrbuch der Europäischen Integration 2011, Baden-Baden 2011, S. 101-112, hier S. 108.

52 Wulf Reiners / Wolfgang Wessels, Rivalität und Gleichgewicht in der institutionellen Architektur der EU, aaO. (FN 4), S. 50.

53 Vgl. Selmayr, Europäische Zentralbank, aaO. (FN 50), S. 99.

54 Vgl. Selmayr, Europäische Zentralbank, aaO. (FN 43), S. 119; »The ECB and the euro: Too central a banker? « in The Economist, 7. September 2012.

55 Vgl. u.a. Mark A. Pollack, The Engines of European Integration. Delegation, Agency, and Agenda Setting in the EU, Oxford 2003.

56 Vgl. Giandomenico Majone, »Two Logics of Delegation: Agency and Fiduciary Relations in EU Governance« in: European Union Politics 2, Nr. 1 (2001), S. 103-22. 
die Beteiligung von Institutionen legitimiert, die nationalen Kontrollinstanzen Rechtfertigung schulden. Ein eigenständiger europäischer Legitimationsstrang, so etwa über eine Beteiligung des Europäischen Parlaments an der Konzeption oder Beaufsichtigung der getroffenen Maßnahmen, wurde nicht vorgesehen. Die hervorstechende Rolle des Europäischen Rats in der Formulierung der Krisenreaktionen kann dabei unterschiedlich bewertet werden. Auf der einen Seite vertreten zentrale Akteure die Ansicht, dass lediglich die Staats- und Regierungschefs - unter der Vorgabe der Einstimmigkeit - über ausreichende demokratische Legitimität verfügen, Maßnahmen mit großer Tragweite wie die Ausgabe gemeinschaftlich garantierter Kredite über den ESM oder die weitreichende Abgabe nationaler Budgethoheit im Zuge des Fiskalpakts zu beschließen: »Die Krise hat die Staats- und Regierungschefs dazu gedrängt, größere Verantwortung zu übernehmen, denn letztendlich verfügen sie allein über die demokratische Legitimität, die es Ihnen erlaubt, zu entscheiden. ${ }^{57}$ Auf der anderen Seite betonen Kritiker dieses Vorgehens, dass die in langen Nachtsitzungen in Brüssel getroffenen Entscheidungen des Europäischen Rats von den Bürgern nicht als Produkte demokratischer Deliberation angesehen werden: »Das Ergebnis einer parlamentarisch unzureichend legitimierten Politik wird von den Bürgern als Diktat aus Brüssel empfunden. Den Preis dafür bezahlt die EU als Ganzes: das ist der Nährboden für antieuropäische Ressentiments. «58

\section{Das Europäische Parlament: Am Rande der Krisenpolitik}

Zwar hatte das Europäische Parlament nie einen starken Einfluss auf »historische « Entscheidungen der Einigungsgeschichte, doch konnte es bisher auf einen kontinuierlichen Zuwachs seiner gesetzgeberischen Kompetenzen als Konsequenz solcher Entscheidungen zurückblicken. Im Verlauf der Krise jedoch wurden dem Europäischen Parlament im Gegensatz zu nahezu allen bisherigen Integrationsschritten keine zusätzlichen Kompetenzen übertragen. Im Unterschied etwa zum deutlichen Gewinn an Rechten durch den Vertrag von Lissabon ${ }^{59}$ wird das EP in den entsprechenden Rechtstexten der Krisenmaßnahmen gar nicht (wie im Fall des ESM-Vertrags) oder nur am Rande erwähnt. ${ }^{60}$ Im Vertrag über Stabilität, Koordinierung und Steuerung sieht lediglich der Abschnitt über die »Steuerung des Euro-Währungsgebiets« die Möglichkeit vor, den Präsidenten des Europäischen Parlaments - analog zu den Regelungen des Europäischen Rats - zu Euro-Gipfeln einzuladen, »um gehört zu werden«(Art. 12 Abs. 4).

57 Nicolas Sarkozy, Discours du Président de la République à Toulon, 1. Dezember 2011 (eigene Übersetzung).

58 Martin Schulz, Antrittsrede von Martin Schulz nach seiner Wabl zum Präsidenten des Europäischen Parlaments, 17. Januar 2012.

59 Vgl. Hofmann / Wessels, Der Vertrag von Lissabon, aaO. (FN 6); Jörg Monar, »The European Union's institutional balance of power after the Treaty of Lisbon « in: Europäische Kommission (Hg.), The European Union after the Treaty of Lisbon: Visions of leading policy-makers, academics and journalists, Luxembourg 2011, S. 60-89.

60 Vgl. Andreas Maurer, »Europäisches Parlament « in: Werner Weidenfeld / Wolfgang Wessels (Hg.), Jabrbuch der Europäischen Integration 2012, Baden-Baden 2012, S. 53-64, hier S. 53.

ZfP 60. Jg. 2/2013 
Generell bemängelte Parlamentspräsident Martin Schulz im Januar 2012 das Vorgehen der Staats- und Regierungschefs, zentrale Maßnahmen außerhalb der EU-Vertragstexte entworfen und damit die Einflussmöglichkeiten des EP geschwächt zu haben: $\$ 99 \%$ aller Regelungen hätte man auch im normalen Gesetzgebungsverfahren erreichen können «. ${ }^{61}$ Tatsächlich konnte das Europäische Parlament Einfluss auf diejenigen Maßnahmen ausüben, die als EU-Gesetzgebungsakte erlassen wurden - also insbesondere das sogenannte »Six-Pack « und das »Two-Pack «. ${ }^{62} \mathrm{Im}$ Zuge der Verhandlungen zwischen EP und Rat über das »Two-Pack « konnte das Europäische Parlament neben einer Verstärkung der parlamentarischen Überwachung der Kommission seine Forderung durchsetzen, der Kommission die Einrichtung einer Expertengruppe aufzutragen, die die Ausgabe gemeinsamer Schuldtitel in Form eines »Europäischen Tilgungsfonds « und EuroAnleihen prüfen soll. ${ }^{63}$ Ansonsten hat das Europäische Parlament mit seinen Forderungen zur Einführung von Eurobonds, einer europäischen Ratingagentur und Wachtumsinitiativen bisher wenig Gehör gefunden. ${ }^{64}$ Dies liegt nicht zuletzt auch daran, dass das EP lediglich über stark begrenzte finanzielle Ressourcen und legislative Zuständigkeiten verfügen kann, die zur Beilegung der Krise dienlich wären.

\section{Nationale Parlamente: Ein nachhaltiges Rückzugsgefecht}

Wichtigste Legitimationsquelle für viele der getroffenen Maßnahmen bleibt daher die nationale Ebene, insbesondere basierend auf der Legitimität nationaler Parlamentsentscheidungen - entweder mittelbar über das Agieren parlamentarisch gestützter und kontrollierter Regierungen, oder direkt über das Instrument der Ratifikation. Letzteres traf insbesondere auf solche Maßnahmen zu, die wie der Fiskalpakt außerhalb der institutionellen Architektur der EU angesiedelt wurden, oder wie der ESM-Vertrag zusätzlich einer Änderung der EU-Verträge bedurften. Beide Maßnahmen mussten den Parlamenten der Teilnehmerstaaten zur Ratifikation nach nationalen Vorgaben vorgelegt werden. Im Fall Irlands war zur Umsetzung des Fiskalpakts zusätzlich eine Volksabstimmung notwendig, bei der sich am 31. Mai 2012 ca. 60\% der Abstimmenden für die Unterzeichnung aussprachen.

Zwar sahen keine der bisherigen Maßnahmen eine rechtliche Fixierung zusätzlicher Kompetenzen nationaler Parlamente in Entscheidungsfindungsprozessen auf der europäischen Ebene vor, der Ratifizierungprozess von ESM-Vertrag und Fiskalpakt führte jedoch in einigen Mitgliedstaaten zu Forderungen nach engerer parlamentarischer Su-

61 Martin Schulz, »Ein europäischer Fiskalpakt ohne parlamentarische Kontrolle ist inakzeptabel! «, Erste Rede von Martin Schulz auf dem Gipfel der EU-Staats- und Regierungschefs, 30. Januar 2012.

62 Vgl. Andreas Maurer, »Europäisches Parlament « in: Werner Weidenfeld / Wolfgang Wessels (Hg.), Jabrbuch der Europäischen Integration 2011, Baden-Baden 2011, S. 53-63, hier S. 53-54.

63 Europäisches Parlament, Grünes Licht für das »Two-Pack"zur wirtschaftspolitischen Stenerung, Pressemitteilung vom 12. März 2013.

64 Martin Schulz, »Ein europäischer Fiskalpakt ohne parlamentarische Kontrolle ist inakzeptabel! «, aaO. (FN 61). 
pervision. ${ }^{65}$ Dabei ist zu beobachten, dass die Diskussion um einen Zuwachs an Kompetenzen nationaler Parlamente in diesem Feld nicht immer von den Parlamenten selbst ausgingen. So betonte etwa das deutsche Bundesverfassungsgericht wiederholt die Parlamentshoheit als eine durch die Krisenreaktionen bedrohte demokratische Grundnorm, nachdem der Bundestag bereits die entsprechenden Maßnahmen verabschiedet hatte: »Als Repräsentanten des Volkes müssen die gewählten Abgeordneten des Deutschen Bundestages auch in einem System intergouvernementalen Regierens die Kontrolle über grundlegende haushaltspolitische Entscheidungen behalten. ${ }^{66}$

Die Rolle nationaler Parlamente als wichtige Legitimationsinstanz kam auch in der Diskussion um ein separates Parlament für die Eurozone zur Sprache. So schlug etwa der ehemalige deutsche Außenminister Joschka Fischer eine »beratende Euro-Kammer« vor, »in die aus den nationalen Parlamenten die Spitzen der Fraktionen und des Parlaments entsandt werden $\ll .{ }^{67}$ Gleichzeitig darf aber nicht übersehen werden, dass die vermehrten Eingriffsrechte der Kommission in die Formulierung nationaler Haushalte in erster Linie auf Kosten der Hoheitsrechte nationaler Parlamente erfolgt, ohne dass diesem Vorgehen ein verstärkte demokratische Kontrolle der Kommission etwa durch das Europäische Parlament oder eine neu zu formende »Euro-Kammer « gegenüber gestellt wurde.

\section{In der Gesamtsicht: Komplexität als Merkmal des Wandels}

In dem Versuch einer Beantwortung der durch die Krise aufgeworfenen Fragen haben die Regierungen der Mitgliedstaaten als zentrale Entscheidungsträger ein komplexes Arsenal an Maßnahmen beschlossen, das die institutionelle Architektur der Europäischen Union nachhaltig verändern wird. Ausgangspunkt der Entscheidungssituation war die Frage, ob die Union überhaupt als adäquate Problemlösungsebene zur Beilegung der durch die drohende Zahlungsunfähigkeit Griechenlands eingeleiteten Krise in Betracht gezogen werden sollte, zumal die ursprüngliche Konzeption der Wirtschafts- und Währungsunion ein Eingreifen nicht vorsah, zum Teil sogar explizit ausschloss. Diese Frage wurde vom weitaus größten Teil der mitgliedstaatlichen Regierungen bejaht. Ihrem Problemlösungsinstinkt folgend verlagerten sie in der Folge weitreichende Kompetenzen im Bereich der Wirtschafts- und Finanzpolitik auf die europäische Ebene. Gleichzeitig entschied sich eine Minderheit von Mitgliedstaaten, Teile dieser Maßnahmen nicht zu übernehmen. Die Krise verstärkt also bestehende Ansätze eines »Europa der mehreren Ge-

65 Vgl. Oliver Höing, »Differentiation of parliamentary powers. The German Constitutional Court and the German Bundestag within the financial crisis « in: Marta Cartabia / Nicola Lupo / Andrea Simoncini (Hg.), Democracy and Subsidiarity in the EU. National parliaments, regions and civil society in the decision-making process, Rome 2013, S. 255-280; Nicolaus Heinen / Alessandro Theiss, EFSF - Where do national parliaments have a say in the EMU?, Deutsche Bank Research, 27. Oktober 2012.

66 Bundesverfassungsgericht, Urteil vom vom 7. September 2011, 2 BvR 987/10, Leitsatz 2b.

67 Joschka Fischer, »Vergesst die EU« in Die Zeit vom 10. November 2011. Vgl. auch Maurer, Europäisches Parlament, aaO. (FN 60), S. 63-64.

ZfP 60. Jg. 2/2013 
schwindigkeiten«, wobei der »Kern« der »integrationswilligen « Staaten eine deutliche Magnetwirkung auf die sich darum gruppierenden Staaten ausübt.

In der Ausgestaltung der Krisenbewältigungsmaßnahmen demonstrierten die Staatsund Regierungschefs einen ausgeprägten »Letztentscheidungsvorbehalt«, indem sie alle wichtigen Leitentscheidungen dem Europäischen Rat unter der Vorgabe der Einstimmigkeit vorbehielten. Auf der Suche nach Effizienz in der Durchführung der beschlossenen Maßnahmen übertrugen sie jedoch der Kommission weitreichende neue Kompetenzen, insbesondere in der Überwachung nationaler Ausgabenpolitik. Weiterhin zeigte sich eine unvorhergesehene Konsequenz der bereits zwei Jahrzehnte zurückliegenden Entscheidung, die gemeinsame Geldpolitik einer unabhängigen Zentralbank übertragen. Die EZB übernimmt in der Krise eine weitaus einflussreichere politische Rolle, als ihr Mandat zur Gewährleistung der Preisstabilität vermuten ließe. Die horizontale Aufgabenverteilung zwischen den Organen der EU zeigt sich als Folge der Krise tiefgreifend verändert.

Zur Rechtfertigung ihrer weitreichenden Entscheidungen zur Bewältigung der Krise verweisen die Staats- und Regierungschefs auf ihre Position als gewählte Vertreter ihrer Bevölkerungen. Demokratische Legitimität beanspruchen sie vor allem über die nationalen Parlamente, die sie stützen, und die einen Teil der Maßnahmen direkt ratifizierten. In diesem Prozess der Ratifikation gelang es einigen nationalen Parlamenten, zusätzliche Rechte in der Überwachung von Entscheidungen auf der EU-Ebene zu erlangen. Gleichzeitig wird die Hoheit nationaler Parlamente über nationale Haushaltspolitik jedoch durch die neuen Vorgaben erheblich eingeschränkt. Das Europäische Parlament wurde nicht als Legitimationsinstanz herangezogen. Ihm wurde entgegen bestehenden Trends keine zentrale Rolle in der zukünftigen Architektur der Wirtschafts- und Währungsunion zugewiesen.

\section{Zusammenfassung}

Existenzkrisen sind Auslöser und Verstärker von grundsätzlichen Verschiebungen interinstitutioneller Beziehungen in allen politischen Systemen. Dies gilt in besonderem Maße für die Europäische Union und die Krise ihrer Wirtschafts- und Währungsunion. Ziel dieses Artikels ist es, diesen Verschiebungen im institutionellen Rahmen der Europäischen Union im Zuge der Krise nachzugehen. Der Artikel stellt dabei drei zentrale Fragen, die die Analyse strukturieren. Wie verändern die Krisenbewältigungsmaßnahmen die (vertikale) Zuständigkeitsverteilung zwischen den Mitgliedstaaten und der Union? Wie verändern die Krisenreaktionen das (horizontale) Gleichgewicht der Aufgabenverteilung zwischen den Akteuren in der institutionellen Architektur der EU? Welche Quellen demokratischer Legitimation beanspruchen die Entscheidungsträger für die neuen Maßnahmen? Unsere Analyse konstatiert dabei die Fortsetzung bereits bestehender Trends sowie einige überraschende Zäsuren. 


\title{
Summary
}

Existential crises are causes and amplifiers of fundamental shifts in inter-institutional relations in all political systems. This is especially true for the European Union and the crisis of Economic and Monetary Union. The aim of this article is to investigate these shifts in the institutional framework of the European Union as the crisis progresses. The article poses three central questions that organise the analysis. How does the crisis response change the vertical division of competences between the member states and the Union? How do the measures taken alter the horizontal balance of power among the institutions of the EU? What sources of democratic legitimacy do the decision-makers claim? Our analysis points to the continuation of existing trends and some surprising breaks.

Andreas Hofmann / Wolfgang Wessels, Tectonic shifts - The crisis as a cause and amplifier of change

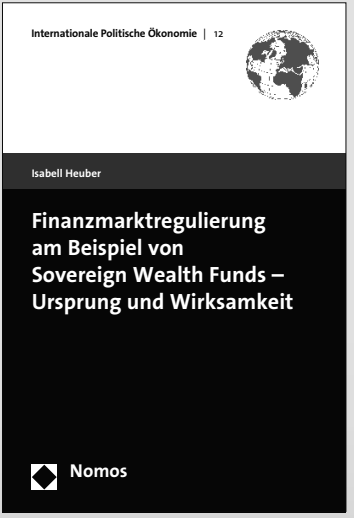

\section{Finanzmarktregulierung am Beispiel von Sovereign Wealth Funds - Ursprung und Wirksamkeit}

\author{
Von Isabell Heuber \\ 2013, 166 S., brosch., 29,- $€$ \\ ISBN 978-3-8487-0537-5 \\ (Internationale Politische Ökonomie, Bd. 12)
}

Der Band untersucht die Regulierung von Sovereign Wealth Funds (SWFs) in den USA und Deutschland. Investitionen von SWFs werden in beiden Ländern trotz sonstiger Marktöffnungsprozesse reguliert. Ursachen und Wirkungsmechanismen dieser Regulierungen liegen in der Einflussnahme partikularistischer Interessen sowie spezifischen institutionellen Kontexten begründet.

Bestellen Sie jetzt telefonisch unter 07221/2104-37. Portofreie Buch-Bestellungen unter www.nomos-shop.de/21045

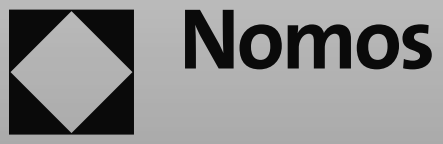

